\title{
Genomic analyses of clonal isolates provide clues to the evolution of Streptococcus pneumoniae
}

\author{
Albert W. Lee ${ }^{1}$, Hervé Tettelin ${ }^{1}$ and Scott Chancey ${ }^{2,3 *}$ \\ Institute for Genome Sciences, University of Maryland School of Medicine, Baltimore, MD, USA \\ 2 Division of Infectious Diseases, Department of Medicine, Emory University School of Medicine, Atlanta, GA, USA \\ ${ }^{3}$ Department of Veterans Affairs Medical Center, Atlanta, GA, USA \\ *Correspondence: scott.chancey@emory.edu
}

\section{A commentary on}

Rapid pneumococcal evolution in response to clinical interventions

by Croucher, N. J., Harris, S. R., Fraser, C., Quail, M. A., Burton, J., van der Linden, M., McGee, L., von Gottberg, A., Song, J. H., Ko, K. S., Pichon, B., Baker, S., Parry, C. M., Lambertsen, L. M., Shahinas, D., Pillai, D. R., Mitchell, T. J., Dougan, G., Tomasz, A., Klugman, K. P., Parkhill, J., Hanage, W. P., and Bentley, S. D. (2011). Science 331, 430-434. doi: 10.1126/science.1198545

The landmark paper recently published by Croucher et al. (2011) reports the draft genome sequencing of 240 Streptococcus pneumoniae isolates and demonstrates the power of comparative genomics in the epidemiological and phylogenetic analysis of bacterial pathogens.

Until recently, the focus of bacterial genome projects has been individual strains or small numbers of clinical isolates. However, with the advent of next generation sequencing platforms such as Roche 454 and Illumina, bacterial genomes can be inexpensively and rapidly sequenced. The first large-scale genomic sequencing project to utilize these technologies for a similar purpose (also by the Bentley group) analyzed 63 clonally related methicillinresistant Staphylococcus aureus isolates (Harris et al., 2010). Croucher et al. (2011) analyzed temporally and geographically diverse isolates of a single $S$. pneumoniae lineage, the multidrug resistant Spain $23 \mathrm{~F}$ ST81 (PMEN1) pandemic clone, to provide insights into the microevolution of this important pathogen.

Streptococcus pneumoniae is a major worldwide public health concern. It is responsible for an estimated 1.6 million deaths each year, half of which are children under the age of five (World Health
Organization, 2003). Efforts to limit pneumococcal disease have focused primarily on antibiotic intervention and vaccination. However, after initial, and often dramatic, successes the effectiveness of these measures has consistently been overcome by the extremely adaptable and resilient pneumococcus. S. pneumoniae is naturally competent and readily incorporates DNA fragments derived from pneumococcal and non-pneumococcal bacterial species into its genome by homologous recombination. High levels of recombination have resulted in extreme diversity within pneumococcal populations from which variant genotypes can rapidly emerge in the presence of changing selective pressures. The primary pressures outside of natural immunity are antibiotics and pneumococcal conjugate polysaccharide vaccines. Resistant and multidrug resistant pneumococci have emerged since the late 1960s and now severely limit treatment options for pneumococcal diseases (Reinert, 2009). The incidences of invasive pneumococcal disease (IPD) and antibiotic resistant pneumococci decreased dramatically following the introduction of the heptavalent conjugate vaccine PCV7 in 2000 (Dagan, 2009). However, the incidence of IPD and resistance is again rising due to the emergence of multidrug resistant, non-vaccine "replacement" serotypes.

Croucher et al. (2011) documented the "escape" of the PMEN1 clone from PCV7 by indentifying several independent horizontal transfer events that resulted in serotype switching of PMEN1 strains from vaccine serotype $23 \mathrm{~F}$ to non-vaccine serotypes. This suggested strong selective pressures for capsule switch variants exerted by the PCV7-immunized human immune system. Evidence for the selective pressures exerted by antibiotics was also reported. Analysis of integrative and con- jugative elements revealed rapid degeneration of such elements but with consistent retention of determinants of resistance to antibiotics commonly used in treatment of upper respiratory infections (e.g., $\beta$-lactams and macrolides). It should be expected that the pneumococcus will adapt quickly to new antibiotic pressures and vaccines such as the 13 -valent PCV vaccine (PCV13) introduced in the United States early 2010. This emphasizes the importance of surveillance and epidemiology to track the emergence of new clones and variations.

In addition to overcoming clinical interventions, the plasticity of the pneumococcal genome has confounded epidemiological analyses. Multilocus sequence typing (MLST) is currently the preferred method for comparing relatedness of isolates within a given bacterial species, and relies on sequence comparison of seven highly conserved alleles. However, the high rate of recombination in pneumococci greatly reduces the resolution of MLST-derived pneumococcal phylogenies due to horizontal acquisition of single nucleotide polymorphisms (SNPs) by recombination events involving MLST alleles. Croucher et al. (2011) avoided this problem by removing horizontally inherited sequences from the phylogenetic analyses. Recombination events were identified by comparison of each genome to a Spain 23F ST81 reference genome (ATCC 700669; Croucher et al., 2009). The phylogeny constructed using only vertically inherited SNPs proved to be a superior estimation of the evolution of the clone based on the correlation between the distance from the root of the tree and the date of isolation of each strain. By comparing the more accurate phylogeny to the dates and locations of isolation, the authors were able to 
pinpoint the likely origins of different clades and to discern information about the geographic spread of the clone.

Future studies utilizinglarge-scalegenomic comparison in S. pneumoniae will likely focus on the influence of antibiotic and vaccine pressures and on the evolution of pneumococcal populations. Whereas Croucher et al. (2011) utilized "deep" sequencing into a single lineage, "wide" genomic analysis of isolates that encompass entire pneumococcal populations will provide insights into the evolution and interactions of multiple lineages. In addition, it will elucidate the effects of localized factors such as host population genetic composition and confounding health and socioeconomic factors. In particular, it will clarify the effects of vaccine and antibiotic pressures on the population and identify candidate lineages for deep sequencing.

Sequencing technologies have also enabled analysis of entire bacterial populations in individual niches, such as the Human Microbiome Project (HMP). The HMP attempts to elucidate the composition and role of the microbiota that inhabit the human body. Such studies in the nasopharynx will provide a better understanding of the role of pneumococcal carriage in invasive disease as well as inter- and intraspecies horizontal gene transfer in S. pneumoniae. Similarly, study of the evolution of pneumococcal isolates within a single host will help define risks of acquired antibiotic resistance in the presence of antibiotic therapy and other interventions.

Current and next generation technologies will be invaluable for epidemiologists and microbiologists studying non-pneumococcal pathogens, and commensals. Large-scale genomic analyses may lead to identification of novel therapeutic agents and new vaccine targets. Croucher et al. (2011) are one of the first groups to realize the full potential of the developments in DNA sequencing technologies that powered these large scale comparative genomic projects. This report signals the beginning of an era of discovery that will lead to comprehensive understanding of bacterial evolution, will revolutionize epidemiology, and allow the development of more effective and longer lasting strategies to treat infectious diseases.

\section{REFERENCES}

Croucher,N.J., Walker,D., Romero,P.,Lennard,N.,Paterson, G.K., Bason, N.C., Mitchell,A.M., Quail,M.A.,Andrew, P.W., Parkhill, J., Bentley, S. D., and Mitchell, T.J. (2009).
Role of conjugative elements in the evolution of the multidrug-resistant pandemic clone Streptococcus pneumoniae Spain23F ST81. J. Bacteriol. 191, 1480-1489.

Dagan, R. (2009). Impact of pneumococcal conjugate vaccine on infections caused by antibiotic-resistant Streptococcus pneumoniae. Clin. Microbiol. Infect. 15, 16-20.

Harris, S. R., Feil, E. J., Holden, M. T. G., Quail, M. A., Nickerson, E. K., Chantratita, N., Gardete, S., Tavares, A., Day, N., Lindsay, J. A., Edgeworth, J. D., De Lencastre, H., Parkhill, J., Peacock, S. J., and Bentley, S. D. (2010). Evolution of MRSA during hospital transmission and intercontinental spread. Science 327, 469-474.

Reinert, R. R. (2009). The public health ramifications of pneumococcal resistance. Clin. Microbiol. Infect. 15, 1-3. World Health Organization. (2003). Weekly Epidemiological Record. Geneva: World Health Organization. 78,97-120.

Received: 22 March 2011; accepted: 23 March 2011; published online: 04 April 2011.

Citation: Lee AW, Tettelin H and Chancey S (2011) Genomic analyses of clonal isolates provide clues to the evolution of Streptococcus pneumoniae. Front. Microbio. 2:63. doi: 10.3389/fmicb.2011.00063

This article was submitted to Frontiers in Cellular and Infection Microbiology, a specialty of Frontiers in Microbiology.

Copyright $(2011$ Lee, Tettelin and Chancey. This is an openaccess article subject to a non-exclusive license between the authors and Frontiers Media SA, which permits use, distribution and reproduction in other forums, provided the original authors and source are credited and other Frontiers conditions are complied with. 